Journal of Mathematics and Statistics 5 (3):171-177, 2009

ISSN 1549-3644

(C) 2009 Science Publications

\title{
The Algebraic K-Theory of Finitely Generated Projective Supermodules P(R) Over a Supercommutative Super-Ring $R$
}

\author{
Ameer Jaber \\ Department of Mathematics, the Hashemite University, Zarqa 13115, Jordan
}

\begin{abstract}
Problem statement: Algebraic K-theory of projective modules over commutative rings were introduced by Bass and central simple superalgebras, supercommutative super-rings were introduced by many researchers such as Knus, Racine and Zelmanov. In this research, we classified the projective supermodules over (torsion free) supercommutative super-rings and through out this study we forced our selves to generalize the algebraic K-theory of projective supermodules over (torsion free) supercommutative super-rings. Approach: We generalized the algebraic K-theory of projective modules to the super-case over (torsion free) supercommutative super-rings. Results: we extended two results proved by Saltman to the supercase. Conclusion: The extending two results, which were proved by Saltman, to the supercase and the algebraic K-theory of projective supermodules over (torsion free) supercommutative super-rings would help any researcher to classify further properties about projective supermodules.
\end{abstract}

Key words: Projective supermodules, superinvolutions, brauer groups, brauer-wall groups

\section{INTRODUCTION}

An associative super-ring $\mathrm{R}=\mathrm{R}_{0}+\mathrm{R}_{1}$ is nothing but a $\mathbb{Z}_{2}$-graded associative ring. A $\mathbb{Z}_{2}$-graded ideal $\mathrm{I}=\mathrm{I}_{0}+\mathrm{I}_{1}$ of an associative super-ring is called a superideal of $R$. An associative super-ring $R$ is simple if it has no non-trivial superideals. Let $\mathrm{R}$ be an associative super-ring with $1 \in \mathrm{R}_{0}$ then $\mathrm{R}$ is said to be a division super-ring if all nonzero homogeneous elements are invertible, i.e., every $0 \neq \mathrm{r}_{\alpha} \in \mathrm{R}_{\alpha}$ has an inverse $\mathrm{r}_{\alpha}^{-1}$, necessarily in $R_{a}$. If $R=R_{0}+R_{1}$ is an associative superring, a (right) $\mathrm{R}$-supermodule $\mathrm{M}$ is a right $\mathrm{R}$-module with a grading $M=M_{0}+M_{1}$ as $R_{0}$-modules such that $\mathrm{m}_{\alpha} \mathrm{r}_{\beta} \in \mathrm{M}_{\alpha+\beta}$ for any $\mathrm{m}_{\alpha} \in \mathrm{M}_{\alpha}, \mathrm{r}_{\beta} \in \mathrm{R}_{\beta}, \alpha, \beta \in \mathbb{Z}_{2}$. An Rsupermodule $M$ is simple if $M R \neq\{0\}$ and $M$ has no proper subsupermodule. Following ${ }^{[4]}$ we have the following definition of R-supermodule homomorphism. Suppose $\mathrm{M}$ and $\mathrm{N}$ are $\mathrm{R}$-supermodules. An Rsupermodule homomorphism from $\mathrm{M}$ into $\mathrm{N}$ is an $\mathrm{R}_{0^{-}}$ module homomorphism $\mathrm{h}_{\gamma}: \mathrm{M} \rightarrow \mathrm{N}, \gamma \in \mathbb{Z}_{2}$, such that $\mathrm{M}_{\alpha} \mathrm{h}_{\gamma} \subseteq \mathrm{N}_{\alpha+\gamma}$. Let $\mathrm{K}$ be a field of characteristic not 2 . An associative superalgebra is a $\mathbb{Z}_{2}$-graded associative $\mathrm{K}$-algebra $\mathrm{A}=\mathrm{A}_{0}+\mathrm{A}_{1}$. A superalgebra $\mathrm{A}$ is central simple over $\mathrm{K}$, if $\hat{\mathrm{Z}}(\mathrm{A})=\mathrm{K}$, where $(\hat{\mathrm{Z}}(\mathrm{A}))_{\alpha}=\left\{\alpha_{\alpha} \in\right.$ $\left.\mathrm{A}_{\alpha}: \alpha_{\alpha} \mathrm{b}_{\beta}=(-1)^{\alpha \beta} \mathrm{b}_{\beta} \alpha_{\alpha} \forall \beta_{\beta} \in \mathrm{A}_{\beta}\right\}$ and the only superideals of $A$ are $(0)$ and $A$. Through out this study we let $R$ be a supercommutative super-ring $(\hat{Z}(A)=R)$ with $1 \in \mathrm{R}_{0}$. An R-superalgebra $\mathrm{A}=\mathrm{A}_{0}+\mathrm{A}_{1}$ is called projective $\mathrm{R}$-supermodule if it is projective as a module over R. Define the superalgebra $A^{e}=A^{o} \widehat{\otimes}_{R} A$, then $A$ is right $A^{e}$-supermodule. There is a natural map $\pi$ from $\mathrm{A}^{\mathrm{e}}$ to A given by deleting o,s and multiplying.

$\operatorname{In}^{[2]}$, Childs, Garfinkel and Orzech proved some results about finitely generated projective supermodules over $\mathrm{R}$, where $\mathrm{R}$ is a commutative ring. $\mathrm{In}^{[1]}$, we generalized the same results about finitely generated projective supermodules over $R$, where $R$ is a supercommutative super-ring. Here are the results:

Proposition 1: Let $\mathrm{M}$ be an R-supermodule and $\mathrm{A}$ an $\mathrm{R}$-superalgebra then there exist isomorphisms of $\mathrm{R}$ superalgebras:

$$
A \widehat{\otimes}_{R} \operatorname{End}_{R}(M) \cong \operatorname{End}_{R}(M) \widehat{\otimes}_{R} A
$$

Corollary 1: Let $\mathrm{P}$ and $\mathrm{Q}$ be a finitely generated projective supermodules over $\mathrm{R}$, then:

$\operatorname{End}_{R}(P) \widehat{\otimes}_{R} \operatorname{End}_{R}(\mathrm{Q}) \cong \operatorname{End}_{R}(\mathrm{Q}) \widehat{\otimes}_{\mathrm{R}} \operatorname{End}_{\mathrm{R}}(\mathrm{P}) \cong$ $\operatorname{End}_{R}\left(\mathrm{P} \widehat{\otimes}_{\mathrm{R}} \mathrm{Q}\right)$

Theorem 1: Let A be an R-superalgebra. The following conditions are equivalent:

- $\mathrm{A}$ is projective right $\mathrm{A}^{\mathrm{e}}$-supermodule

- $0 \mapsto \operatorname{ker}(\pi) \mapsto \mathrm{A}^{\mathrm{e}} \stackrel{\pi}{\longrightarrow} \mathrm{A} \rightarrow 0$ splits as a sequence of right $\mathrm{A}^{\mathrm{e}}$-supermodules 
- $\left(\mathrm{A}^{\mathrm{e}}\right)_{0}$ contains an element $\varepsilon$ such that $\pi(\varepsilon)=1$ and $\varepsilon\left(1 \otimes \mathrm{a}_{\alpha}\right)=\varepsilon\left(\mathrm{a}_{\alpha} \otimes 1\right)$ for all $\mathrm{a}_{\alpha} \in \mathrm{A}_{\alpha}$

Definition 1: We say that A is R-separable if conditions (1-3) above hold.

\section{Remarks:}

- Condition (3) states that A is R-separable if and only if it is R-separable of the sense of ungraded algebras

- It is easy to see that $\varepsilon$ defined above is idempotent. $\mathrm{A}$ is a central separable R-superalgebra if it is separable as an R-algebra, thus our Azumaya Ralgebras A are those separable R-algebras which are superalgebras over $\mathrm{R}$ and whose supercenter is $\mathrm{R}$

For any R-superalgebra $\mathrm{A}$ we have seen that $\mathrm{A}$ is naturally a right $A^{\mathrm{e}}$-supermodule. This induces an $\mathrm{R}$ superalgebra homomorphism $\mu$ from $A^{e}$ to $\operatorname{End}_{R}(A)$ by associating to any element $x_{\alpha} \otimes y_{\beta}$ of $A^{e}$ the element $\mathrm{x}_{\alpha} \mathrm{y}_{\beta}$ where for any $\mathrm{a}_{\gamma} \in \mathrm{A}_{\gamma}$ :

$$
\mathrm{a}_{\gamma} \mu\left(\mathrm{x}_{\alpha} \otimes \mathrm{y}_{\beta}\right)=\mathrm{a}_{\gamma} \cdot\left(\mathrm{x}_{\alpha} \mathrm{y}_{\beta}\right)=(-1)^{\alpha \gamma} \mathrm{x}_{\alpha} \mathrm{a}_{\gamma} \mathrm{y}_{\beta}
$$

Theorem 2: Let A be an R-superalgebra. The following conditions are equivalent:

- A is an Azumaya R-superalgebra

- $\mathrm{A}$ is finitely generated faithful projective $\mathrm{R}$ supermodule and $\mu$ is an isomorphism

\section{MATERIALS AND METHODS}

Suppose $\mathrm{C}$ is any category and $\operatorname{obj}(\mathrm{C})$ the class of all objects of $\mathrm{C}$ and let $\mathrm{C}(\mathrm{A}, \mathrm{B})$ be the set of all morphisms $\mathrm{A} \rightarrow \mathrm{B}$, where $\mathrm{A}, \mathrm{B} \in \operatorname{obj}(\mathrm{C})$. A groupoid is a category in which all morphisms are isomorphisms.

Definition 2: A category with product is a groupoid $\mathrm{C}$, together with a product functor $\perp: \mathrm{C} \times \mathrm{C} \rightarrow \mathrm{C}$ which is assumed to be associative and commutative.

A functor $\mathrm{F}:(\mathrm{C}, \perp) \rightarrow\left(\mathrm{C}^{\prime}, \perp^{\prime}\right)$ of categories with product is a functor $\mathrm{F}: \mathrm{C} \rightarrow \mathrm{C}^{\prime}$ which preserves the product.

\section{Examples:}

- Let $\mathrm{R}$ be any supercommutative super-ring and let $\mathrm{P}(\mathrm{R})$ denote the category of finitely generated projective supermodules over Rwith isomorphisms as morphisms. It is a category with product if we set $\perp=\oplus$

- The subcategory $\mathrm{FP}(\mathrm{R})$ of $\mathrm{P}(\mathrm{R})$ with finitely generated faithful projective supermodules as objects. Her we set $\perp=\widehat{\otimes}_{\mathrm{R}}$

- The category $A z(R)$ of Azumaya superalgebras over R. Her we take $\perp=\widehat{\otimes}_{\mathrm{R}}$

If $\mathrm{C}(\mathrm{R})$ denotes one of the categories mentioned above and if $R \rightarrow R^{\prime}$ is a homomorphism of super-rings. Then $\mathrm{R}^{\prime} \widehat{\otimes}_{\mathrm{R}}$ induces a functor $\mathrm{C}(\mathrm{R}) \rightarrow \mathrm{C}^{\prime}\left(\mathrm{R}^{\prime}\right)$ preserving product.

Definition 3: Let $\mathrm{C}$ be a category with product. The Grothendieck group of $\mathrm{C}$ is defined to be an abelian group $\mathrm{K}_{0} \mathrm{C}$, together with the map ( $)_{\mathrm{C}}$ : $\operatorname{obj}(\mathrm{C}) \rightarrow \mathrm{K}_{0} \mathrm{C}$, which is universal for maps into abelian groups satisfying:

- $\quad$ if $A \cong B$, then $(A)_{C}=(B)_{C}$

- $(\mathrm{A} \perp \mathrm{B})_{\mathrm{C}}=(\mathrm{A})_{\mathrm{C}}+(\mathrm{B})_{\mathrm{C}}$

Definition 4: A composition on a category $(\mathrm{C}, \perp)$ is a composition of objects of $\mathrm{C}$, which satisfies the following condition: if $\mathrm{A} \circ \mathrm{A}^{\prime}$ and $\mathrm{B} \circ \mathrm{B}^{\prime}$ are defined then so also is $(\mathrm{A} \perp \mathrm{B}) \circ\left(\mathrm{A}^{\prime} \perp \mathrm{B}^{\prime}\right)$ and:

$$
(\mathrm{A} \perp \mathrm{B}) \circ\left(\mathrm{A}^{\prime} \perp \mathrm{B}^{\prime}\right)=\left(\mathrm{A} \circ \mathrm{A}^{\prime}\right) \perp\left(\mathrm{B} \circ \mathrm{B}^{\prime}\right)
$$

Definition 5: If $(C, \perp, \circ)$ is a category with product and composition. Then the Grothendieck group of $\mathrm{C}$ is defined to be an abelian group $\mathrm{K}_{0} \mathrm{C}$, together with a map:

$$
()_{\mathrm{C}}: \operatorname{obj}(\mathrm{C}) \rightarrow \mathrm{K}_{0} \mathrm{C}
$$

which is universal for maps into abelian groups satisfying the two conditions in Definition 3 and:

If $\mathrm{A} \circ \mathrm{B}$ is defined, then $(\mathrm{A} \circ \mathrm{B})_{\mathrm{C}}=(\mathrm{A})_{\mathrm{C}}+(\mathrm{B})_{\mathrm{C}}$

An easy computation gives us the following result.

Proposition 2: Let $(\mathrm{C}, \perp, \circ)$ be a category with product and composition. Then:

- Every element of $\mathrm{K}_{0} \mathrm{C}$ has the form $(\mathrm{A})_{\mathrm{C}}-(\mathrm{B})_{\mathrm{C}}$ for some A, B in obj(C)

- $(A)_{C}=(B)_{C}$ if and only if $\exists C, D_{0}, D_{1}, E_{0}, E_{1} \in$ $\operatorname{obj}(\mathrm{C})$, such that $\mathrm{D}_{0}{ }^{\circ} \mathrm{D}_{1}$ and $\mathrm{E}_{0}{ }^{\circ} \mathrm{E}_{1}$ are defined and $\mathrm{A} \perp \mathrm{C} \perp\left(\mathrm{D}_{0} \circ \mathrm{D}_{1}\right) \perp \mathrm{E}_{0} \perp \mathrm{E}_{1} \cong \mathrm{B} \perp \mathrm{C} \perp \mathrm{D}_{0} \perp \mathrm{D}_{1} \perp\left(\mathrm{E}_{0} \circ \mathrm{E}_{1}\right)$ 
- If $\mathrm{F}: \mathrm{C} \rightarrow \mathrm{C}^{\prime}$ is a functor of categories with product and composition, then $\mathrm{F}$ preserves the composition. Moreover, the map $\mathrm{K}_{0} \mathrm{~F}: \mathrm{K}_{0} \mathrm{C} \rightarrow \mathrm{K}_{0} \mathrm{C}^{\prime}$ given by $(\mathrm{A})_{\mathrm{C}}$ $\rightarrow(\mathrm{FA})_{\mathrm{C}^{\prime}}$ is well-defined and makes $\mathrm{K}_{0} \mathrm{~F}$ a functor into abelian groups

Now let $(C, \perp)$ be a groupoid. For $A \in \operatorname{obj}(C)$, we write $\mathrm{G}(\mathrm{A})=\mathrm{C}(\mathrm{A}, \mathrm{A})$, the group of automorphisms of A. If $\mathrm{f:} \mathrm{A} \rightarrow \mathrm{B}$ is an isomorphism, then we have a homomorphism $\mathrm{G}(\mathrm{f}): \mathrm{F}(\mathrm{A}) \rightarrow \mathrm{G}(\mathrm{B})$, given by $G(f)(\alpha)=f \alpha f^{-1}$.

We shall construct, out of $\mathrm{C}$, a new category $\Omega \mathrm{C}$. we take $\operatorname{obj}(\Omega \mathrm{C})$ to be the collection of all automorphisms of C. If $\alpha \in \operatorname{obj}(\Omega C)$ is an automorphism of $A \in C$, we write $(A, \alpha)$ instead of $\alpha$. A morphism $(\mathrm{A}, \alpha) \rightarrow(\mathrm{B}, \beta)$ in $\Omega \mathrm{C}$ is a morphism $\mathrm{f}: \mathrm{A} \rightarrow \mathrm{B}$ in $\mathrm{C}$ such that the diagram in Fig. 1 is commutative, that is $G(f)(\alpha)=\beta$. The product in $\Omega C$ is defined by setting $(\mathrm{A}, \alpha) \perp(\beta, \beta)=(\mathrm{A} \perp \mathrm{B}, \alpha \perp \beta)$. The natural composition $\circ$ is defined in $\Omega \mathrm{C}$ as follows: if $\alpha, \beta \in \operatorname{obj}(\Omega C)$ are automorphisms of the same object in $C$, then $\alpha \circ \beta=\alpha \beta$ and:

$$
(\alpha \perp \beta) \circ\left(\alpha^{\prime} \perp \beta^{\prime}\right)=\alpha \alpha^{\prime} \perp \beta \beta^{\prime}
$$

Definition 6: If $(C, \perp)$ is a category with product, we define:

$$
\mathrm{K}_{1} \mathrm{C}=\mathrm{K}_{0} \Omega \mathrm{C}
$$

If $\mathrm{F}: \mathrm{C} \rightarrow \mathrm{C}^{\prime}$ is a functor, then $\Omega \mathrm{F}: \Omega \mathrm{C} \rightarrow \Omega \mathrm{C}^{\prime}$, preserving product and composition, so we obtain homomorphisms $\mathrm{K}_{\mathrm{i}} \mathrm{F}: \mathrm{K}_{\mathrm{i}} \mathrm{C} \rightarrow \mathrm{K}_{\mathrm{i}} \mathrm{C}^{\prime}, \mathrm{i}=0,1$.

If $\mathrm{P}(\mathrm{R})$ is the category of finitely generated projective R-supermodules, where $R$ is a supercommutative super-ring and their isomorphisms with $\oplus$. Then the tensor product $\widehat{\otimes}_{\mathrm{R}}$ is additive with respect to $\oplus$ so that it induces on $\mathrm{K}_{0} \mathrm{P}(\mathrm{R})$ a structure of commutative ring.

The next following results are just the generalizing of the results proved by $\mathrm{H}$. Bass to the supercase.

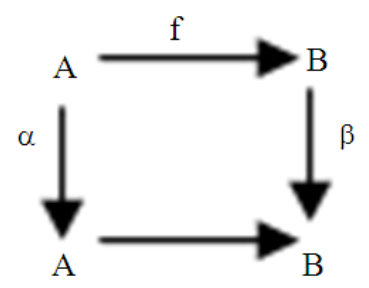

Fig. 1: Set of morphisms
If $\mathrm{Z} \in \operatorname{spec}(\mathrm{R})$ (i.e., $\mathrm{Z} \subseteq \mathrm{R}$ is a prime superideal) and $\mathrm{P} \in \mathrm{P}(\mathrm{R})$, then $\mathrm{P}_{\mathrm{Z}}$ is a free $\mathrm{R}_{\mathrm{Z}}$-supermodule and its rank is denoted by $\mathrm{rk}_{\mathrm{P}}(\mathrm{Z})$. The map:

$$
\mathrm{rk}_{\mathrm{p}}: \operatorname{spec}(\mathrm{R}) \rightarrow \mathbb{Z}
$$

given by $\mathrm{Z} \rightarrow \mathrm{rk}_{\mathrm{P}}(\mathrm{Z})$ is continuous and is called the rank of $\mathrm{P}$. As $\mathrm{R}$ is a supercommutative super-ring, $\mathrm{K}_{0}$ $\mathrm{P}(\mathrm{R})$ and $\mathrm{Q} \widehat{\otimes}_{\mathrm{R}} \mathrm{K}_{0} \mathrm{P}(\mathrm{R})=\mathrm{QK}_{0} \mathrm{P}(\mathrm{R})$ are rings with multiplication induced by $\widehat{\otimes}_{R}$. Since:

$$
\mathrm{rk}_{\mathrm{P} \oplus \mathrm{Q}}=\mathrm{rk}_{\mathrm{P}}+\mathrm{rk}_{\mathrm{Q}}
$$

and

$$
\mathrm{rk}_{\mathrm{P} \oplus_{\mathrm{R}} \mathrm{Q}}=\mathrm{rk}_{\mathrm{P}} \mathrm{rk}_{\mathrm{Q}}
$$

We have a rank homomorphism:

$$
\mathrm{rk}_{\mathrm{P}}: \mathrm{K}_{0} \mathrm{P}(\mathrm{R}) \rightarrow \mathrm{C}
$$

where $\mathrm{C}$ is the ring of continuous functions $\operatorname{spec}(\mathrm{R}) \rightarrow \mathbb{Z}$.

The rank homomorphism rk is splitting by the ring homomorphism $\mathrm{C} \rightarrow \mathrm{K}_{0} \mathrm{P}(\mathrm{R})$, so that:

$$
\mathrm{K}_{0} \mathrm{P}(\mathrm{R}) \cong \mathrm{C} \oplus \widetilde{\mathrm{K}_{0}} \mathrm{P}(\mathrm{R})
$$

where, $\widetilde{\mathrm{K}_{0}} \mathrm{P}(\mathrm{R})=\operatorname{ker}(\mathrm{rk})$ So:

$$
\mathbb{Q} \otimes_{\mathbb{Z}} \mathrm{K}_{0} \mathrm{P}(\mathrm{R}) \cong\left(\mathbb{Q} \otimes_{\mathbb{Z}} \mathrm{C}\right) \oplus\left(\mathbb{Q} \otimes_{\mathbb{Z}} \widetilde{\mathrm{K}_{0}} \mathrm{P}(\mathrm{R})\right)
$$

The next results generalize the results proved by $\mathrm{H}$. Bass.

Theorem 3: Suppose $\max (\mathrm{R})$, the space of maximal superideals of $\mathrm{R}$, is noetherian space of dimension $\mathrm{d}$, then:

- If $x \in \mathrm{K}_{0} \mathrm{P}(\mathrm{R})$ and $\operatorname{rk}(\mathrm{x}) \geq \mathrm{d}$, then $\mathrm{x}=(\mathrm{p})_{\mathrm{P}(\mathrm{R})}$ for some $P \in P(R)$

- If $\operatorname{rk}\left((\mathrm{P})_{\mathrm{P}(\mathrm{R})}\right)>\mathrm{d}$ and if $\left(\mathrm{P}_{\mathrm{P}(\mathrm{R})}\right)=\left(\mathrm{Q}_{\mathrm{P}(\mathrm{R})}\right)$, then $\mathrm{P} \approx \mathrm{Q}$

- $\left(\widetilde{\mathrm{K}_{0}} \mathrm{P}(\mathrm{R})\right)^{\mathrm{d}+1}=0$

Proposition 3: The following conditions on Rsupermodule $\mathrm{P}$ are equivalent:

- $\quad \mathrm{P}$ is a finitely generated projective supermodule over $\mathrm{R}$ and has zero ahnihlator

- $\quad \mathrm{P} \in \mathrm{P}(\mathrm{R})$ and has every where positive rank 
- $\quad \exists$ a supermodule $\mathrm{Q}$ and a positive integer $\mathrm{n}$ such that $\mathrm{P} \widehat{\otimes}_{\mathrm{R}} \mathrm{Q} \approx \mathrm{R}^{\mathrm{n}}$

\section{RESULTS AND DISCUSSION}

Let $\mathrm{P}(\mathrm{R})$ be the category of finitely generated projective supermodules over $\mathrm{R}, \mathrm{Az}(\mathrm{R})$ the category of Azumaya superalgebras over $R$ and $\operatorname{Prog}(R)$ the category of finitely generated faithful projective Rsupermodules.

A useful fact to be remember is that since $\mathrm{R}$ is supercommutative super-ring, $\mathrm{P} \in \operatorname{Prog}(\mathrm{R})$ if and only if $P \in \operatorname{Prog}(R)$ and $P$ is faithful. If $A, B \in A z(R)$ are equivalent in $B W(R)$ (the Brauer-Wall group of $R$ ), we will write $A \sim B$. If $M$ is a supermodule over $R$, then $\mathrm{nM}$ is the $\mathrm{n}$-fold direct sum of $\mathrm{M}$. If $\mathrm{P} \in \mathrm{P}(\mathrm{R})$ let $(\mathrm{P})$ be the image of $\mathrm{P}$ in $\mathrm{K}_{0} \mathrm{P}(\mathrm{R})$ and $\{\mathrm{P}\}$ in $\mathbb{Q} \otimes_{\mathbb{Z}} \mathrm{K}_{0} \mathrm{P}(\mathrm{R})=\mathbb{Q}$ $\mathrm{K}_{0} \mathrm{P}(\mathrm{R})$. The next results generalize the results proved by $^{[6]}$.

Theorem 4: Let $P, P^{\prime}, Q \in P(R)$. Then:

- $\quad \mathrm{P} \in \operatorname{Prog}(\mathrm{R})$ if and only if there is a $\mathrm{Q}$ in $\mathrm{P}(\mathrm{R})$ such that $\mathrm{P} \widehat{\otimes}_{\mathrm{R}} \mathrm{Q}$ is free

- If $\mathrm{x} \in \mathbb{Q} \mathrm{K}_{0} \mathrm{P}(\mathrm{R})$ and $\operatorname{rk}(\mathrm{x})>0$ then $\mathrm{x}=\left(\frac{1}{\mathrm{~m}}\right)\{\mathrm{Q}\}$ for some $Q \in \operatorname{Prog}(R), m>0$ an integer

- If $\{P\}=\{Q\}, P \in \operatorname{Prog}(R)$, then there is an integer $\mathrm{n}>0$ such that $\mathrm{nP} \approx \mathrm{nQ}$

- If $\mathrm{Q} \in \operatorname{Prog}(\mathrm{R})$ and $\left((\mathrm{P})-\left(\mathrm{P}^{\prime}\right)\right)(\mathrm{Q})=0$ then there is an integer $\mathrm{n}>0$ such that $\mathrm{nP} \approx \mathrm{nP}^{\prime}$

- If $P \in \operatorname{Prog}(R)$ and $r k_{P}$ is a square then there is an integer $\mathrm{n}>0$ and $\mathrm{Q} \in \operatorname{Prog}(\mathrm{R})$ such that $\mathrm{n}^{2} \mathrm{P} \approx \mathrm{Q} \widehat{\otimes}_{\mathrm{R}} \mathrm{Q}$

Let $\mathrm{R} / \mathrm{S}$ be Galois extension of supercommutative super-rings with finite Galois Group G. $M=M_{0}+M_{1}$, an R-supermodule, has a $\mathrm{G}$-action if there is a group injection $\varphi: G \rightarrow \operatorname{Aut}(M)$ such that $\varphi(\sigma)$ is $\sigma$-linear for all $\sigma \in \mathrm{G}$. That is, $\varphi(\sigma)\left(\mathrm{m}_{\alpha} \mathrm{r}_{\beta}\right)=\varphi(\sigma)\left(\mathrm{m}_{\alpha}\right) \sigma\left(\mathrm{r}_{\beta}\right)$. Let $M^{G}=\{m \in M: \varphi(\sigma)(m)=m$ for all $\sigma \in G\}$. The following fact was proved in ${ }^{[1]}$, if $M \in \operatorname{Prog}(R), M^{G} \operatorname{Prog}(S)$ then:

$$
\mathrm{R} \widehat{\otimes}_{\mathrm{S}} \mathrm{M}^{\mathrm{G}} \cong \mathrm{M}
$$

Again let R/S be a Galois extension of supercommutative super-rings with Galois group $G=\{1, \sigma\}$. Let $\mathrm{A}$ be any central separable R-superalgebra, we define $A^{\sigma}$ as follows, set $A^{\sigma}=A$ as a super-ring, but the product by a scalar. on $A^{\sigma}$ is defined by $\lambda \mathrm{a}=\sigma(\lambda) \mathrm{a}$ for all $\lambda \in R$. Then one easily check that $A^{\sigma}$ is a central separable R- superalgebra.

Now let $\tau: A^{\sigma} \widehat{\otimes}_{R} A \rightarrow A^{\sigma} \widehat{\otimes}_{R} A$, be defined by $\tau\left(\mathrm{a}_{\alpha} \otimes \mathrm{b}_{\beta}\right)=(-1)^{\alpha \beta} \mathrm{b}_{\beta} \otimes \mathrm{a}_{\alpha}$, then $\tau$ is a $\sigma$-linear automorphism. In particular $\tau$ is S-linear. Define the Corestriction:

$$
\operatorname{Tr}(\mathrm{A})=\left\{\mathrm{x} \in \mathrm{A}^{\sigma} \widehat{\otimes}_{\mathrm{R}} \mathrm{A} \mid \tau(\mathrm{x})=\mathrm{x}\right\}
$$

Obviously, $\operatorname{Tr}(\mathrm{A})$ is an S-superalgebra. But by ${ }^{[3]} \operatorname{Tr}(\mathrm{A})$ is an S-progenerator as an S-supermodule, if $\mathrm{A}$ is an R-progenerator as an R-supermodule. Moreover if $A$ is central separable over $R$ then $b^{\left[{ }^{[3]}\right.} \operatorname{Tr}(A)$ is central separable over S.

Lemma 1: Let $\mathrm{R} / \mathrm{S}$ be a Galois extension of supercommutative super-rings with Galois group $G=\{1, \sigma\}$. Let A, B be R-supermodules (superalgebras), $\mathrm{P} \in$ $\operatorname{Prog}(\mathrm{R})$ :

- If $\mathrm{A}$ and $\mathrm{B}$ have $\mathrm{G}$-action, so does $\mathrm{M}=\mathrm{A} \widehat{\otimes}_{\mathrm{R}} \mathrm{B}$ and $\mathrm{M}^{\mathrm{G}}=\mathrm{A}^{\mathrm{G}} \otimes_{\mathrm{R}} \mathrm{B}^{\mathrm{G}}$

- $\operatorname{Tr}\left(\mathrm{A} \widehat{\otimes}_{\mathrm{R}} \mathrm{B}\right) \cong \operatorname{Tr}(\mathrm{A}) \widehat{\otimes}_{\mathrm{S}} \operatorname{Tr}(\mathrm{B})$

- $\quad$ If $E=\operatorname{End}_{R}(P), \operatorname{Tr}(E) \cong \operatorname{End}_{S}(\operatorname{Tr}(P))$

Theorem 5: Let $A \in A z(R)$ and $P, Q \in \operatorname{Prog}(A)$ such that $\mathrm{P} \approx \mathrm{Q}$ as $\mathrm{R}$-supermodules. Then there is an integer $\mathrm{n}>0$ such that $\mathrm{nP} \approx \mathrm{nQ}$ as A-supermodules.

Proof: $\quad \mathrm{A} \widehat{\otimes}_{\mathrm{R}} \operatorname{End}_{\mathrm{A}}(\mathrm{P}) \cong \operatorname{End}_{\mathrm{R}}(\mathrm{P}) \cong \operatorname{End}_{\mathrm{R}}(\mathrm{Q}) \cong \mathrm{A} \widehat{\otimes}_{\mathrm{R}}$ $\operatorname{End}_{A}(Q)$. Tensoring by $A^{\circ}$ yields that:

$$
\operatorname{End}_{\mathrm{R}}(\mathrm{A}) \otimes_{\mathrm{R}} \operatorname{End}_{\mathrm{A}}(\mathrm{P}) \cong \operatorname{End}_{\mathrm{R}}(\mathrm{A}) \otimes_{\mathrm{R}} \operatorname{End}_{\mathrm{A}}(\mathrm{Q})
$$

or

$$
\operatorname{End}_{\mathrm{A}}\left(\mathrm{A} \otimes_{\mathrm{R}} \mathrm{P}\right) \cong \operatorname{End}_{\mathrm{A}}\left(\mathrm{A} \otimes_{\mathrm{R}} \mathrm{Q}\right)
$$

where, $A$ acts on $A \widehat{\otimes}_{R} P\left(A \widehat{\otimes}_{R} Q\right)$ by acting on $P(Q)$. Using $^{[3]}$, There is a rank one projective R-supermodule I, such that $\mathrm{A} \widehat{\otimes}_{\mathrm{R}} \mathrm{P} \cong \mathrm{A} \widehat{\otimes}_{\mathrm{R}} \mathrm{Q} \widehat{\otimes}_{\mathrm{R}} \mathrm{I}$ as $\mathrm{A}$-supermodules. Theorem 4(a) implies that $m R \widehat{\otimes}_{R} P \cong m R \widehat{\otimes}_{R} Q \widehat{\otimes}_{R} I$ as A-supermodules, for some $m>0$ and $m^{\prime} R \cong m^{\prime} R \widehat{\otimes}_{R} I$ as R-supermodules, for some different $\mathrm{m}^{\prime}$. Finally, $\mathrm{n}=\mathrm{mm}^{\prime}$ will satisfy the theorem.

On a superalgebra $\mathrm{A}$, a map $\mathrm{J}: \mathrm{A} \rightarrow \mathrm{A}$ is called a superinvolution if $\mathrm{J}^{2}$ is the identity and $\mathrm{J}$ is an 
antiautomorphism. More explicitly, $\left(\mathrm{a}_{\alpha}\right)^{\mathrm{J}^{2}}=\mathrm{a}_{\alpha}$, $\left(a_{\alpha}+b_{\beta}\right)^{J}=a_{\alpha}^{J}+b_{\beta}^{J}$ and $\left(a_{\alpha} b_{\beta}\right)^{J}=(-1)^{\alpha \beta} b_{\beta}^{J} a_{\alpha}^{J}$ for all $a_{\alpha}, b_{\beta} \in A$. Let $C=\hat{Z}(A)$ (the super-center of A) then $J$ must preserve $C$. If $\mathrm{J}$ is the identity on $\mathrm{C}, \mathrm{J}$ is a superinvolution of the first kind. If not, $\mathrm{J}$ induces an automorphism of $\mathrm{C}$ of order 2 and $\mathrm{J}$ is said to be of the second kind. Two superinvolutions $\mathrm{J}, \mathrm{J}^{\prime}$ which agree on $\mathrm{C}$ are said to be of the same kind.

The following theorem generalizes of ${ }^{[6]}$.

Theorem 6: If $A \in A z(R)$ and $A \widehat{\otimes}_{R} A \sim 1$, then there is a $\mathrm{B} \in \mathrm{Az}(\mathrm{R})$, such that $\mathrm{B} \sim \mathrm{A}$ and $\mathrm{B} \cong \mathrm{B}^{\circ}$.

Another way of viewing an isomorphism $B \cong B^{\circ}$ is that $\mathrm{B}$ has an antiautomorphism, J, of the first kind. Now, we are ready to prove the following result.

Theorem 7: Suppose A is a super-ring with antiautomorphism $\mathrm{J}$ such that $\mathrm{J}^{2}$ is inner, induced by a $\mathrm{w}_{0} \in \mathrm{A}_{0}$ such that $\mathrm{w}_{0}\left(\mathrm{w}_{0}\right)^{\mathrm{J}}=\left(\mathrm{w}_{0}\right)^{\mathrm{J}} \mathrm{w}_{0}=1$. Then $\mathrm{M}_{2}(\mathrm{~A})$ has a superinvolution of the same kind.

Proof Let L be the inverse map to J. Since:

$$
\mathrm{w}_{0}^{-1} \mathrm{a}_{\alpha} \mathrm{w}_{0}=\left(\mathrm{a}_{\alpha}\right)^{\mathrm{J}^{2}}
$$

We have $\left(\mathrm{a}_{\alpha}\right)^{\mathrm{J}}\left(\mathrm{w}_{0}\right)^{\mathrm{J}}=\left(\mathrm{w}_{0}\right)^{\mathrm{J}}\left(\mathrm{a}_{\alpha}\right)^{\mathrm{L}} \quad$ and $\left(\mathrm{a}_{\alpha}\right)^{\mathrm{L}} \mathrm{w}_{0}=\mathrm{w}_{0}\left(\mathrm{a}_{\alpha}\right)^{\mathrm{J}}$, so the map:

$$
\left(\begin{array}{cc}
\mathrm{a}_{\alpha} & \mathrm{b}_{\alpha} \\
\mathrm{c}_{\alpha} & \mathrm{d}_{\alpha}
\end{array}\right) \mapsto\left(\begin{array}{cc}
\left(\mathrm{d}_{\alpha}\right)^{\mathrm{J}} & \left(\mathrm{w}_{0}\right)^{\mathrm{J}}\left(\mathrm{b}_{\alpha}\right)^{\mathrm{L}} \\
\mathrm{w}_{0}\left(\mathrm{c}_{\alpha}\right)^{\mathrm{J}} & \left(\mathrm{a}_{\alpha}\right)^{\mathrm{L}}
\end{array}\right)
$$

Is a superinvolution on $\mathrm{M}_{2} \mathrm{~A}$ of the same kind of $\mathrm{J}$.

Next we try to find the conditions on a central separable R-superalgebra $\mathrm{A}$ to have a superinvolution of the second kind, if $\mathrm{R}$ is a connected super-ring. In the next theorem we try to find the conditions on $A=\operatorname{End}_{R}(P)$ to have a superinvolution of any kind, where $\mathrm{P}$ is an R-progenerator as a supermodule over $\mathrm{R}$, if $\mathrm{R}$ is a connected super-ring.

The following theorem involves assuming that $\mathrm{R}$, the base super-ring, is semilocal. We will use the fact, from $^{[5]}$, that if $\mathrm{A}, \mathrm{B}$ are central separable R-algebras, $\mathrm{A} \sim \mathrm{B}$ and the rank of $\mathrm{A}$ equals $\operatorname{rank}$ of $\mathrm{B}$, then $\mathrm{A} \cong \mathrm{B}$, which is also true in the superalgebra case (i.e., if $A, B$ are central separable R-superalgebras, $\mathrm{A} \sim \mathrm{B}$ and the rank of $A$ equals rank of $B$, then $A \cong B$ ). Let $M$ be the Jacobson radical of $\mathrm{R}$. Then $\overline{\mathrm{A}}=\mathrm{A} / \mathrm{MA}$ is a finite direct sum of simple superalgebras. We call $\bar{A}$ is perfect if every simple subsuperalgebra of $\overline{\mathrm{A}}$ admits a superinvolution of the second kind.

Theorem 8: Suppose $\mathrm{R}$ is a connected semilocal superring and $\mathrm{A}$ is a central separable R-superalgebra. Suppose $\mathrm{R} / \mathrm{S}$ is a Galois extension with Galois group $\{1, \sigma\}$. Then $\mathrm{A}$ has a superinvolution of the second kind extending $\sigma$ if and only if $\operatorname{Tr}(\mathrm{A}) \sim 1$ and $\overline{\mathrm{A}}$ is perfect.

Proof: Suppose A has a superinvolution, *, extending $\sigma$. Then it is easy to Check that $\overline{\mathrm{A}}$ is perfect. Also * induces an isomorphism $\mathrm{A}^{\sigma} \cong \mathrm{A}^{\circ}$, so there is an isomorphism:

$$
\varphi: \mathrm{A}^{\sigma} \widehat{\otimes}_{\mathrm{R}} \mathrm{A} \rightarrow \operatorname{End}_{\mathrm{R}}(\mathrm{A})
$$

given by $\mathrm{x}_{\gamma}\left(\mathrm{a}_{\alpha} \otimes \mathrm{b}_{\beta}\right)^{\varphi}=(-1)^{\alpha \gamma} \mathrm{a}_{\alpha}^{*} \mathrm{x}_{\gamma} \mathrm{b}_{\beta}$. Set $\mathrm{A}^{\prime}=\mathrm{A}_{0}^{\prime}+\mathrm{A}_{1}^{\prime}$, where $\mathrm{A}_{\alpha}^{\prime}=\left\{\mathrm{a}_{\alpha} \in \mathrm{A}_{\alpha}: \mathrm{a}_{\alpha}^{*}=\mathrm{a}_{\alpha}\right\}$.

Since ${ }^{*}$ is $\sigma$-linear $\mathrm{R}$-supermodule automorphism of $\mathrm{A}$, the $\mathrm{S}$-supermodule $\mathrm{A}^{\prime}$ is an $\mathrm{S}$ progenerator as a module over $S . \quad \varphi$ induces an isomorphism $\operatorname{Tr}(\mathrm{A}) \cong \operatorname{End}_{\mathrm{S}}\left(\mathrm{A}^{\prime}\right)$, hence $\operatorname{Tr}(\mathrm{A}) \sim 1$.

Conversely, since $\mathrm{R}$ is a connected semilocal super-ring, one easily sees that $S$ is a connected semilocal super-ring also. Let $\operatorname{Tr}(\mathrm{A}) \cong \operatorname{End}_{\mathrm{s}}(\mathrm{P})$. In other words let $\tau: \mathrm{A}^{\sigma} \widehat{\otimes}_{\mathrm{R}} \mathrm{A} \rightarrow \mathrm{A}^{\sigma} \widehat{\otimes}_{\mathrm{R}} \mathrm{A}$ given by $\left(\mathrm{a}_{\alpha} \otimes \mathrm{b}_{\beta}\right)^{\tau}=(-1)^{\alpha \beta} \mathrm{b}_{\beta} \otimes_{\mathrm{a}_{\alpha}}$, be a $\sigma$-linear automorphism. Then $\operatorname{Tr}(\mathrm{A})$ is the fixed super-ring of $\mathrm{A}^{\sigma} \widehat{\otimes}_{\mathrm{R}} \mathrm{A}$ under $\tau$. Say $\operatorname{Tr}(\mathrm{A}) \cong \operatorname{End}_{\mathrm{S}}(\mathrm{P})$, where Pis an S-progenerator as a supermodule over $\quad \mathrm{S}$. Then $\mathrm{A}^{\sigma} \widehat{\otimes}_{R} \mathrm{~A} \cong \mathrm{R} \widehat{\otimes}_{\mathrm{R}} \operatorname{End}_{\mathrm{S}}(\mathrm{P}) \cong \operatorname{End}_{\mathrm{R}}\left(\mathrm{R} \widehat{\otimes}_{\mathrm{S}} \mathrm{P}\right) \quad$ and $\quad$ if $\varphi=\sigma \otimes 1: R \widehat{\otimes}_{S} P \rightarrow R \widehat{\otimes}_{S} P, \quad$ then $\left(\mathrm{x}_{\gamma}\left(\mathrm{a}_{\alpha} \otimes \mathrm{b}_{\beta}\right)\right)^{\varphi}=\mathrm{x}_{\gamma}^{\varphi}\left(\mathrm{a}_{\alpha} \otimes \mathrm{b}_{\beta}\right)^{\tau}, \quad$ for $\quad$ all $\quad \mathrm{x}_{\gamma} \in \mathrm{R} \widehat{\otimes}_{\mathrm{S}} \mathrm{P}$ and $\mathrm{a}_{\alpha} \otimes \mathrm{b}_{\beta} \in \mathrm{A}^{\sigma} \widehat{\otimes}_{\mathrm{R}} \mathrm{A}$. Since $\mathrm{R} \quad$ is connected, $\operatorname{rank}_{R}(A)=\operatorname{rank}_{R}\left(A^{\sigma}\right)$, but:

$$
A^{\sigma} \widehat{\otimes}_{R} A \cong \operatorname{End}_{R}\left(R \widehat{\otimes}_{S} P\right)
$$

Therefore:

$$
A^{\sigma} \widehat{\otimes}_{R}\left(A \widehat{\otimes}_{R} A^{\circ}\right) \cong A^{\sigma} \widehat{\otimes}_{R} \operatorname{End}_{R}(A) \cong \operatorname{End}_{R}\left(R \widehat{\otimes}_{S} P\right) \widehat{\otimes}_{R} A^{\circ}
$$

So $\quad b y^{[5]}, \quad A^{\sigma} \cong A^{\circ}$, which implies that $\operatorname{End}_{R}(\mathrm{~A}) \cong \operatorname{End}_{R}\left(\mathrm{R} \widehat{\otimes}_{S} \mathrm{P}\right)$, but the R-rank of A equals the 
$\mathrm{R}$-rank of $\mathrm{R} \widehat{\otimes}_{\mathrm{S}} \mathrm{P}$. So again by ${ }^{[5]}, \mathrm{A} \cong \mathrm{R} \widehat{\otimes}_{\mathrm{S}} \mathrm{P}$. In other words, $\mathrm{A}$ has a $\sigma$-linear antiautomorphism $\mathrm{J}$ such that for all $\mathrm{a}_{\alpha}, \mathrm{x}_{\gamma}, \mathrm{b}_{\beta}$ in A, setting $\mathrm{x}_{\gamma}\left(\mathrm{a}_{\alpha} \otimes \mathrm{b}_{\beta}\right)=(-1)^{\alpha \lambda} \mathrm{a}_{\alpha}^{\mathrm{J}} \mathrm{x}_{\gamma} \mathrm{b}_{\beta}$ yields the isomorphism $\mathrm{A}^{\sigma} \otimes_{\mathrm{R}} \mathrm{A} \cong \operatorname{End}_{\mathrm{R}}(\mathrm{A})$ and the map $\varphi: \sigma \otimes 1: \mathrm{A}\left(\cong \mathrm{R} \widehat{\otimes}_{\mathrm{S}} \mathrm{P}\right) \rightarrow \mathrm{A}$ satisfies $\quad \varphi^{2}=1 \quad$ and $\left(\mathrm{x}_{\gamma} \cdot\left(\mathrm{a}_{\alpha} \otimes \mathrm{b}_{\beta}\right)\right)^{\varphi}=\mathrm{x}_{\gamma}^{\varphi} \cdot\left(\mathrm{a}_{\alpha} \otimes \mathrm{b}_{\beta}\right)^{\tau}$. Therefore:

$$
(-1)^{\alpha \lambda}\left(a_{\alpha}^{J} x_{\gamma} b_{\beta}\right)^{\varphi}=(-1)^{\alpha \beta} x_{\gamma}^{\varphi} \cdot\left(b_{\beta} \otimes a_{\alpha}\right)=(-1)^{\beta(\alpha+\gamma)} b_{\beta}^{J} \mathbf{x}_{\gamma}^{\varphi} a_{\alpha}
$$

( $\varphi$ respects the grading). For $\mathrm{w}=1^{\varphi} \in \mathrm{A}_{0}$ we have $\mathrm{ww}^{\mathrm{J}}=\mathrm{w}^{\mathrm{J}} \mathrm{w}=1$ and $\mathrm{wa}_{\alpha} \mathrm{w}^{-1}=\mathrm{a}_{\alpha}^{\mathrm{J}^{2}}$ and:

$\varphi^{2}=1,\left(\mathrm{a}_{\alpha}^{\mathrm{J}} \mathrm{x}_{\gamma} \mathrm{b}_{\beta}\right)^{\varphi}=(-1)^{\alpha \lambda}(-1)^{\beta(\alpha+\gamma)} \mathrm{b}_{\beta}^{\mathrm{J}} \mathrm{x}_{\gamma}^{\varphi} \mathrm{a}_{\alpha}$

Lemma 2: Let A be a central separable R-superalgebra, with $\mathrm{J}$ and $\varphi$ satisfying (1). Then $\mathrm{A}$ has a superinvolution agreeing with $\mathrm{J}$ on $\mathrm{R}$ if $\varphi$ fixes a unit of $\mathrm{A}_{\mathrm{a}}$.

Proof: If $\mathrm{u}_{\mathrm{a}}$ is a unit in $\mathrm{A}_{\mathrm{a}}$ such that $\mathrm{u}_{\alpha}^{\varphi}=\mathrm{u}_{\alpha}$ then $\mathrm{u}_{\alpha}=\left(1 . \mathrm{u}_{\alpha}\right)^{\varphi}=\mathrm{u}_{\alpha}^{\mathrm{J}} \mathrm{w}, \quad$ so $\quad\left(\mathrm{u}_{\alpha}^{\mathrm{J}}\right)^{-1} \mathrm{u}_{\alpha}=\mathrm{w}, \quad$ but $\left(\mathrm{u}_{\alpha}^{\mathrm{J}}\right)^{-1}=(-1)^{\alpha}\left(\mathrm{u}_{\alpha}^{-1}\right)^{\mathrm{J}}$, therefore $\mathrm{w}=(-1)^{\alpha}\left(\mathrm{u}_{\alpha}^{-1}\right)^{\mathrm{J}} \mathrm{u}_{\alpha}$, implying that $\mathbf{x}_{\gamma}^{j^{\prime}}=\mathrm{u}_{\alpha}^{-1} \mathrm{x}_{\gamma}^{\mathrm{J}} \mathrm{u}_{\alpha}$ is a superinvolution since $\mathrm{J}^{\prime}$ is an antiautomorphism on $\mathrm{A}$ and:

$$
\begin{aligned}
\left(\mathrm{x}_{\gamma}^{\mathrm{j}}\right)^{\mathrm{J}^{\prime}} & =\mathrm{u}_{\alpha}^{-1}\left(\mathrm{u}_{\alpha}^{-1} \mathrm{x}_{\gamma}^{\mathrm{J}} \mathrm{u}_{\alpha}\right)^{\mathrm{J}} \mathrm{u}_{\alpha}=(-1)^{\alpha} \mathrm{u}_{\alpha}^{-1}\left(\mathrm{u}_{\alpha}^{\mathrm{J}} \mathrm{x}_{\gamma}^{\mathrm{J}^{2}}\left(\mathrm{u}_{\alpha}^{-1}\right)^{\mathrm{J}}\right) \mathrm{u}_{\alpha} \\
& =\mathrm{u}_{\alpha}^{-1} \mathrm{u}_{\alpha}^{\mathrm{J}}\left(\mathrm{wx}_{\gamma} \mathrm{w}^{-1}\right) \mathrm{w} \\
& =\mathrm{x}_{\gamma}, \text { since } \mathrm{u}_{\alpha}^{-1} \mathrm{u}_{\alpha}^{\mathrm{J}}=\mathrm{w}^{-1}
\end{aligned}
$$

Continuing proof of the theorem: Let $\mathrm{M}$ be the jacobson radical of $\mathrm{R}$. Then $\overline{\mathrm{A}}=\mathrm{A} / \mathrm{MA}$ is a finite direct sum of simple superalgebras. On $\bar{A}, \varphi$ and $\mathrm{J}$ induce maps $\bar{\varphi}$ and $\overline{\mathrm{J}}$ satisfying (1). Every preimage of a unit $\overline{\mathrm{u}}_{\alpha}$ of $\overline{\mathrm{A}}$ is a unit $\mathrm{u}_{\mathrm{a}}$ of $\mathrm{A}_{\mathrm{a}}$. Thus we can change $\mathrm{J}$ by conjugation with a unit $u_{a}$, to make $\bar{J}$ any desired antiautomorphism of $\overline{\mathrm{A}}$ of the same kind. In fact, if $\mathrm{J}^{\prime}$ is defined by $x_{\gamma}^{j^{\prime}}=u_{\alpha}^{-1} x_{\gamma}^{j} u_{\alpha}$, we can find a corresponding $\varphi$ 'so that $J^{\prime}, \varphi$ ' satisfy (1). Specifically if $L$ is the inverse map to J, we can set $\mathrm{x}_{\gamma}^{\varphi}=\mathrm{u}_{\alpha}^{-1} \mathbf{x}_{\gamma}^{\varphi} \mathrm{u}_{\alpha}^{\mathrm{L}}$, to show that we have:

$$
\begin{aligned}
\left(\mathbf{x}_{\gamma}^{\varphi}\right)^{\varphi^{\prime}} & =\mathrm{u}_{\alpha}^{-1}\left(\mathrm{u}_{\alpha}^{-1} \mathbf{x}_{\gamma}^{\varphi} \mathrm{u}_{\alpha}^{\mathrm{L}}\right)^{\varphi} \mathrm{u}_{\alpha}^{\mathrm{L}} \\
& =(-1)^{\alpha} \mathrm{u}_{\alpha}^{-1}\left(\mathrm{u}_{\alpha}^{\mathrm{L}} \mathrm{x}_{\gamma} \mathrm{z}_{\alpha}\right) \mathrm{u}_{\alpha}^{\mathrm{L}}
\end{aligned}
$$

where $\mathrm{z}_{\alpha}^{\mathrm{J}}=\mathrm{u}_{\alpha}^{-1}$ and hence $\mathrm{z}_{\alpha}=\mathrm{z}_{\alpha}^{\mathrm{JL}}=\left(\mathrm{u}_{\alpha}^{-1}\right)^{\mathrm{L}}$, so that $\left(\mathrm{x}_{\gamma}^{\varphi^{\prime}}\right)^{\phi^{\prime}}=(-1)^{\alpha} \mathrm{x}_{\gamma}\left(\mathrm{u}_{\alpha}^{-1}\right)^{\mathrm{L}} \mathrm{u}_{\alpha}^{\mathrm{L}}=\mathrm{x}_{\gamma}$ since $(-1)^{\alpha}\left(\mathrm{u}_{\alpha}^{\mathrm{L}}\right)^{-1}=\left(\mathrm{u}_{\alpha}^{-1}\right)^{\mathrm{L}}$. It suffices to find $\overline{\mathrm{u}}_{\alpha}$ of $\overline{\mathrm{A}}$ such that $\left(\overline{\mathrm{u}}_{\alpha}\right)^{\varphi}+\overline{\mathrm{u}}_{\alpha}$ is a unit, for if $\mathrm{u}_{\alpha}$ is a preimage of $\overline{\mathrm{u}}_{\alpha}$, then $\left(\mathrm{u}_{\alpha}\right)^{\varphi}+\mathrm{u}_{\alpha}$ will be a $\varphi$ fixed unit of $A_{a}$. Since $\bar{A}$ is perfect, it suffices to prove.

Lemma 3: Let $\bar{A}$ be a finite dimensional central simple superalgebra over a field $F$ with a superinvolution $\mathbf{J}$ of the second kind and any associated $\varphi$ to $\mathrm{J}$ then there is an element $\overline{\mathrm{a}}_{\alpha}$ in $\overline{\mathrm{A}}_{\alpha}$ such that $\left(\overline{\mathrm{a}}_{\alpha}\right)^{\varphi}+\overline{\mathrm{a}}_{\alpha}$ is a unit.

Proof: The element $w=1^{\varphi}$ is central since $J$ is a superinvolution. If $\mathrm{w} \neq-1$, then $\overline{\mathrm{a}}_{0}=1$ will do. If $\mathrm{W}=-1$, then $\left(\overline{\mathrm{a}}_{\alpha}\right)^{\varphi}=\left(\overline{\mathrm{a}}_{\alpha}\right)^{\mathrm{J}} \mathrm{W}=-\left(\overline{\mathrm{a}}_{\alpha}\right)^{\mathrm{J}}$. Since $\mathrm{J}$ is of order 2 on $F$, there is $f$ in $F$ such that $f-f^{J} \neq 0$, so again take $\overline{\mathrm{a}}_{0}=\mathrm{f}-\mathrm{f}^{\mathrm{J}}$.

Lemma 4: Suppose $Q$ is a right $A^{e}=A^{\circ} \widehat{\otimes}_{R} A$ supermodule, then:

$$
\mathrm{Q}=\mathrm{M} \oplus \mathrm{I}
$$

where, $\mathrm{M}$ is the $\mathrm{R}$-subsupermodule of $\mathrm{Q}$ generated by all elements of the form $\left(a_{\alpha} \otimes 1-1 \otimes a_{\alpha}\right) q_{\beta}$, where $\mathrm{a}_{\alpha} \in \mathrm{A}_{\alpha}$ and $\mathrm{q}_{\beta} \in \mathrm{Q}_{\beta}$. If $\mathrm{Q}$ is R-projective as a supermodule over $\mathrm{R}$ then:

$\operatorname{rank}_{R}(A) \cdot \operatorname{rank}_{R}(I)=\operatorname{rank}_{R}(Q)$.

Proof: Consider the well-known split exact sequence of $\mathrm{A}^{\mathrm{e}}$-supermodules:

$$
0 \rightarrow \mathrm{J} \rightarrow \mathrm{A}^{\mathrm{e}} \stackrel{\mu}{\longrightarrow} \mathrm{A} \rightarrow 0
$$

where, $\mu\left(a_{\alpha} \otimes b_{\beta}\right)=a_{\alpha} b_{\beta}$ and $J$ is a right super-ideal of $A^{\mathrm{e}}$ generated by all elements of the form $\mathrm{a}_{\alpha} \otimes 1-1 \otimes \mathrm{a}_{\alpha}$ where $\mathrm{a}_{\alpha} \in \mathrm{A}_{\alpha}$. Suppose $\mathrm{Q}$ is a right $\mathrm{A}^{\mathrm{e}}$-supermodule. Tensoring by $\mathrm{Q}$ over $\mathrm{A}^{\mathrm{e}}$ yields a split exact sequence of R-supermodules:

$$
0 \rightarrow \mathrm{Q} \widehat{\otimes}_{\mathrm{A}^{\circ}} \mathrm{J} \rightarrow \mathrm{Q} \widehat{\otimes}_{\mathrm{A}^{\circ}} \mathrm{A}^{\mathrm{e}} \stackrel{1 \otimes \mu}{\longrightarrow} \mathrm{Q} \widehat{\otimes}_{\mathrm{A}^{\circ}} \mathrm{A} \rightarrow 0
$$

of course, $\mathrm{Q} \widehat{\otimes}_{\mathrm{A}^{\mathrm{e}}} \mathrm{A}^{\mathrm{e}} \cong \mathrm{Q}$ under the map $\mathrm{a}_{\alpha} \otimes \mathrm{z}_{\beta} \mapsto \mathrm{a}_{\alpha} \mathrm{z}_{\beta}$. Under this isomorphism $\mathrm{Q} \widehat{\otimes}_{\mathrm{A}^{0}} \mathrm{~J}$ is mapped onto $\mathrm{M}$ defined above. Thus $\mathrm{Q} \cong \mathrm{M} \oplus \mathrm{I}$, where $\mathrm{I} \cong \mathrm{Q} \widehat{\otimes}_{\mathrm{A}^{\circ}} \mathrm{A}$. But: 


$$
I \widehat{\otimes}_{R} A \cong Q \widehat{\otimes}_{A^{e}}\left(A \widehat{\otimes}_{R} A\right) \cong Q \widehat{\otimes}_{A^{e}}\left(A^{\circ} \widehat{\otimes}_{R} A\right)
$$

as an R-supermodules, therefore, $\mathrm{I} \widehat{\bigotimes}_{\mathrm{R}} \mathrm{A} \cong \mathrm{Q} \widehat{\otimes}_{\mathrm{A}^{\mathrm{e}}} \mathrm{A}^{\mathrm{e}} \cong \mathrm{Q}$.

Suppose $\mathrm{R}$ is a local supercommutative super-ring, $\sigma$ an automorphism of $\mathrm{R}$ of order 2, P is an Rprogenerator as a supermodule over $\mathrm{R}$ and $\mathrm{I}$ a rank one R-projective supermodule. A morphism e $\mathrm{P} \widehat{\bigotimes}_{\mathrm{R}} \mathrm{P} \rightarrow \mathrm{I}$ is called a bilinear I form on $\mathrm{P}$, a morphism e $: \mathrm{P}^{\sigma} \widehat{\otimes}_{\mathrm{R}} \mathrm{P} \rightarrow \mathrm{I}$ is called a $\sigma$ bilinear I form on P. The image $e\left(p_{\alpha} \otimes q_{\beta}\right)$ is often written as $\mathrm{e}\left(\mathrm{p}_{\alpha}, \mathrm{q}_{\beta}\right)$ and in either case, e can be thought of as a map e $: \mathrm{P} \times \mathrm{P} \rightarrow \mathrm{I}$. Such a form induces a map $\mathrm{e}^{*}: \mathrm{P} \rightarrow \operatorname{Hom}_{\mathrm{R}}(\mathrm{P}, \mathrm{I}) \quad\left(\mathrm{P}^{\sigma} \rightarrow \operatorname{Hom}_{\mathrm{R}}(\mathrm{P}, \mathrm{I})\right)$ given by $\mathrm{e}^{*}\left(\mathrm{p}_{\alpha}\right)\left(\mathrm{q}_{\beta}\right)=\mathrm{e}\left(\mathrm{p}_{\alpha}, \mathrm{q}_{\beta}\right)$. In a similar manner, we define $\mathrm{e}_{*}: \mathrm{P} \rightarrow \operatorname{Hom}_{\mathrm{R}}(\mathrm{P}, \mathrm{I}) \quad\left(\mathrm{P} \rightarrow \operatorname{Hom}_{\mathrm{R}}\left(\mathrm{P}^{\sigma}, \mathrm{I}\right)\right) \quad$ given by $\mathrm{e}_{*}\left(\mathrm{p}_{\alpha}\right)\left(\mathrm{q}_{\beta}\right)=\mathrm{e}\left(\mathrm{p}_{\alpha}, \mathrm{q}_{\beta}\right)$. If $\mathrm{e}^{*}$ and $\mathrm{e}_{*}$ are isomorphisms then we say $\mathrm{e}$ is nondegenerate. The next final result shows that the existence of superinvolutions on $\operatorname{End}_{\mathrm{R}}(\mathrm{p})$, where $\operatorname{End}_{\mathrm{R}}(\mathrm{p})$ is an R-progenerator as a supermodule over $\mathrm{R}$, is equivalent to the existence of forms on $\mathrm{P}$ and this result was proved in ${ }^{[1]}$.

Theorem 9: Let $\mathrm{R}$ be a connected super-ring and $\mathrm{A}=\operatorname{End}_{\mathrm{R}}(\mathrm{p})$ be a central separable R-superalgebra such that $\mathrm{A}$ is an R-progenerator as a supermodule over $\mathrm{R}$, then:

- A has a superinvolution of the first kind if and only if there is a rank one R-projective $\mathrm{I}$, a nondegenerate bilinear I form e on $\mathrm{P}$ and a $\delta \in \mathrm{R}_{0}$ such that $\delta^{2}=1$ and $\mathrm{e}\left(\mathrm{x}_{\alpha}, \mathrm{y}_{\beta}\right)=(-1)^{\alpha \beta} \delta \mathrm{e}\left(\mathrm{y}_{\beta}, \mathrm{x}_{\alpha}\right)$ for all $\mathrm{x}_{\alpha}, \mathrm{y}_{\beta}$ in $\mathrm{P}$

- Let $\sigma$ be an automorphism of R of order 2. Then A has a superinvolution of the second kind extending $\sigma$ if and only if there is a rank one R-projective I with a $\sigma$-linear automorphism of order 2 (also called $\sigma$ ) a $\sigma$-bilinear I form e on P and an element $\delta$ in $\mathrm{R}_{0}$ such that $\sigma(\delta) \delta=1$ and $\sigma\left(\mathrm{e}\left(\mathrm{x}_{\alpha}, \mathrm{y}_{\beta}\right)\right)=(-1)^{\alpha \beta} \delta \mathrm{e}\left(\mathrm{y}_{\beta}, \mathrm{x}_{\alpha}\right)$ for all $\mathrm{x}_{\alpha}, \mathrm{y}_{\beta}$ in $\mathrm{P}$

\section{CONCLUSION}

The extended two results proved by Saltman ${ }^{[6]}$ to the supercase and the algebraic K-theory of projective supermodules over (torsion free) supercommutative super-rings would help any researcher to classify further properties about projective supermodules.

\section{REFERENCES}

1. Jaber, A., 2003. Superinvolutions of Associative Superalgebras, P.h. D. thesis, University of Ottawa, Ottawa, Canada, 2003.

2. Childs, L.N., G. Garfinkel and M. Orzech, 1973. The brauer group of graded azumaya algebras, Trans. Am. Math. Soc., 175: 299-326. DOI: 10.1007/BFb0077340

3. DeMeyer, F. and E. Ingraham, 1971. Separable Algebras Over Commutative Rings, L.N.M. 181, Springer-Verlag, New York, USA., ISBN: 9783540053712, pp: 172.

4. Racine, M.L., 1998. Primitive superalgebras with superinvolution, J. Algebra, 206: 588-614. DOI: 10.1006/JABR.1997.7412

5. Roy, A. and R. Sridharan, 1967. Derivations in azumaya algebras. Math. Kyot. Univ., 72: 161-167.

6. Saltman, D.J., 1978. Azumaya algebras with involution. J. Algebra, 52: 526-539. DOI: 10.1016/0021-8693(78)90253-3 